\title{
Combined Invariants to Gaussian Blur and Affine Transformation
}

\author{
Jitka Kostková and Jan Flusser \\ Czech Academy of Sciences \\ Institute of Information Theory and Automation \\ Pod vodárenskou věží 4, 18208 Prague 8, Czech Republic \\ Email: kostkova@utia.cas.cz, \\ flusser@utia.cas.cz
}

\author{
Matteo Pedone \\ Center for Machine Vision Research \\ Department of Computer Science and Engineering \\ University of Oulu \\ Oulu FI-90014, Finland \\ Email: matteo.pedone@oulu.fi
}

\begin{abstract}
The paper presents a new theory of combined moment invariants to Gaussian blur and spatial affine transformation. The blur kernel may be arbitrary oriented, scaled and elongated. No prior information about the kernel parameters and about the underlaying affine transform is required. The main idea, expressed by the Substitution Theorem, is to substitute pure blur invariants into traditional affine moment invariants. Potential applications of the new descriptors are in blur-invariant image recognition and in robust template matching.
\end{abstract}

\section{INTRODUCTION}

Invariants to image blurring are an efficient alternative to an ill-posed and time-consuming image restoration/deconvolution whenever a complete restoration of the image is not necessary and can be avoided. A typical example is a recognition of objects in blurred images, where a blur-robust object description forms a sufficient input for the classifier. Roughly speaking, blur invariant $I$ is a functional fulfilling the constraint $I(f)=I(f * h)$ for any $h$ from a certain set $S$ of admissible blurring point-spread functions (PSF's).

In this paper, we concentrate our attention to the case when the PSF is a Gaussian function with unknown parameters and at the same time, the image has undergone an affine distortion. So, our acquisition model is

$$
g(\mathbf{x})=(f * h)(A \mathbf{x}),
$$

where $f$ is an ideal image, $h$ is a Gaussian PSF, $A$ is a regular matrix of spatial transformation, and $g$ denotes the observed image. This linear image formation model, even if it is very simple, is a reasonably accurate approximation of many imaging devices and acquisition scenarios.

The novel contribution of this paper is the design of the combined invariants to Gaussian blur and spatial affine transformation. This problem has not been tackled in the literature so far. Our idea is based on the coupling of recent invariants w.r.t. a general (anisotropic) Gaussian blur [1] with traditional affine moment invariants. The way how to reach the combined invariance is expressed in the Substitution Theorem, which performs the main result of the paper.

\section{RELATED WORK}

Invariants w.r.t. blur were originally proposed in the work by Flusser et al. [2], [3] and have been used and further developed since then by many authors. Individual systems of blur invariants differ from one another by the assumptions imposed on the PSF, by the mathematical tools used for invariant construction, by the domain in which the invariants are defined, and by the application area which the invariants were designed for (see [4], Chapter 6, for a survey of blur invariants and other references).

Only few systems of invariants have been designed specifically for Gaussian blur. Liu and Zhang [5] realized that the complex moments of the image, one index of which is zero, are invariant to Gaussian blur. Xiao [6] seemingly derived invariants to Gaussian blur but in fact he only employed symmetry of the Gaussian rather than its specific form. Höschl proposed invariants to Gaussian convolution in 1D and applied them to image histograms [7]. Flusser et al. [8] introduced a complete set of moment-based Gaussian blur invariants. Zhang et al. [9] proposed implicit invariants, which enable a blur-invariant comparison of two images.

Serious weakness of all above mentioned Gaussian-blur invariant methods is that they assume circularly symmetric Gaussian blur only and cannot be extended to the general case of an arbitrary shaped Gaussian blur. This generalization is not trivial and requires new approaches. This is also the reason why the above methods cannot combine the invariance to blur with the invariance to affine transformation. Under an affine transformation, a circular Gaussian becomes elliptic and the blur invariance property is violated. Since the input image is rarely captured in a spatially rectified position and thus certain geometric deformation is present almost always, this is a critical limitation for a practical usage.

Most recently, Kostková et al. [1] published the first paper ever on invariants w.r.t. Gaussian blur with a non-diagonal covariance matrix but the idea of the combined invariants was not mentioned in that paper. In this paper, we adopt the Gaussian blur invariants from [1] and show how to use them as "building blocks" for the design of combined affine-blur invariants.

\section{GAUSSIAN BLUR}

By an image function (or just image for short) $f(\mathbf{x})$ we understand any function from $L_{1}\left(\mathbb{R}^{2}\right)$, the integral of which 
is nonzero. For the sake of generality, we do not constraint it to be non-negative.

2D Gaussian function $G_{\Sigma}$ is given as

$$
G_{\Sigma}(\mathbf{x})=\frac{1}{2 \pi \sqrt{|\Sigma|}} \exp \left(-\frac{1}{2} \mathbf{x}^{T} \Sigma^{-1} \mathbf{x}\right)
$$

where $\mathbf{x} \equiv(x, y)^{T}$ and $\Sigma$ is a $2 \times 2$ regular covariance matrix. Since the covariance matrix is positive definite, we have, for its determinant, $|\Sigma|>0$.

The set $S$ of all Gaussian blurring kernels is

$$
S=\left\{a G_{\Sigma} \mid a>0, \Sigma \text { positive definite }\right\} .
$$

The set $S$ is closed under convolution, point-wise multiplication, and Fourier transform. This means that the result of these operations, when applied on functions from $S$, is again an element of $S$. In this paper, we particularly use the coordinate transform closure under the transform $\mathrm{x}^{\prime}=A \mathbf{x}$.

Proposition 1. Let $A$ be a regular matrix and let $h(\mathbf{x}) \in S$. Then $h^{\prime}(\mathbf{x}) \equiv h(A \mathbf{x}) \in S$.

This proposition follows from the fact that

$$
a G_{\Sigma}(A \mathbf{x})=\frac{a}{\|A\|} G_{A^{-1} \Sigma A^{-T}}(\mathbf{x})
$$

where $\|A\|$ means the absolute value of the determinant of $A$ and $A^{-T} \equiv\left(A^{T}\right)^{-1}=\left(A^{-1}\right)^{T}$.

\section{RECALLING GAUSSIAN BLUR INVARIANTS}

In this Section, we briefly recall the Gaussian blur invariants from [1] up to the extent which is necessary for understanding their use in the case of compound transformation.

Let us define the projection operator $P$ such that it projects image $f$ onto the nearest un-normalized Gaussian, where the term "nearest" means the Gaussian having the same integral and covariance matrix as the image $f$ itself. So, we define

$$
P f=m_{00} G_{C},
$$

where

$$
C=\frac{1}{m_{00}}\left(\begin{array}{ll}
m_{20} & m_{11} \\
m_{11} & m_{02}
\end{array}\right),
$$

and $m_{p q}$ is the centralized image moment

$$
m_{p q}=\iint\left(x-c_{1}\right)^{p}\left(y-c_{2}\right)^{q} f(x, y) \mathrm{d} x \mathrm{~d} y
$$

with $\left(c_{1}, c_{2}\right)$ being the image centroid.

Operator $P$ commutes with convolution with a Gaussian kernel as

$$
P\left(f * G_{\Sigma}\right)=P f * G_{\Sigma}
$$

Thanks to this,

$$
I(f)=\frac{\mathcal{F}(f)}{\mathcal{F}(P f)}
$$

is an invariant to Gaussian blur, i.e. $I(f)=I(f * h)$ for any $h \in S(\mathcal{F}(f)$ denotes Fourier transform of $f)$.

All three factors in Eq. (7) can be expanded into absolutely convergent Taylor series. We recall that geometric moments of an image are Taylor coefficients (up to a constant factor) of its Fourier transform, so we obtain a relation between the moments of $f, P f$, and $f_{r}=\mathcal{F}^{-1}(I(f))$. Note that $f_{r}$ itself may not exist in $L_{1}$ but working with its moments is correct. If $f_{r}$ exists, it can be understood as the "maximally possible" deconvolved non-Gaussian component of $f$ by the kernel $P f$ and in [1] it is called the primordial image of $f$. Primordial image is obviously a blur invariant, as well as all its moments $M_{p q}$. Comparing the coefficients of the same powers and expressing the moments of $P f$ in terms of the moments of $f$, we obtain the final recurrent relation for Gaussian blur invariants $M_{p q}$ in the moment domain

$$
\begin{aligned}
& M_{p q}=\frac{m_{p q}^{(f)}}{m_{00}}-\sum_{\substack{l=0 \\
l+k \neq 0, l+k \text { even }}}^{p} \sum_{\substack{k=0 \\
l}}^{q}\left(\begin{array}{l}
p \\
l
\end{array}\right)\left(\begin{array}{l}
q \\
k
\end{array}\right) \sum_{\substack{i=0 \\
j \geq \frac{k-l}{2}}}^{\left\lfloor\frac{k}{2}\right\rfloor} \sum_{j=0}^{i}(-1)^{i-j}\left(\begin{array}{c}
k \\
2 i
\end{array}\right)\left(\begin{array}{l}
i \\
j
\end{array}\right) . \\
& \cdot(l+k-2 i-1) ! !(2 i-1) ! !\left(\frac{m_{11}}{m_{00}}\right)^{k-2 j} \text {. } \\
& \cdot\left(\frac{m_{20}}{m_{00}}\right)^{\frac{l-k}{2}+j}\left(\frac{m_{02}}{m_{00}}\right)^{j} M_{p-l, q-k} .
\end{aligned}
$$

Note that the blur invariants $M_{p q}$ can be calculated directly from the moments of $f$, without constructing the projection $P f$ and computing any Fourier transform. Moreover, no prior knowledge of the blurring kernel parameters is required.

The main idea behind the derivation of the blur invariants (8) is depicted in Fig. 1. For the details and complete proofs, we refer to [1].

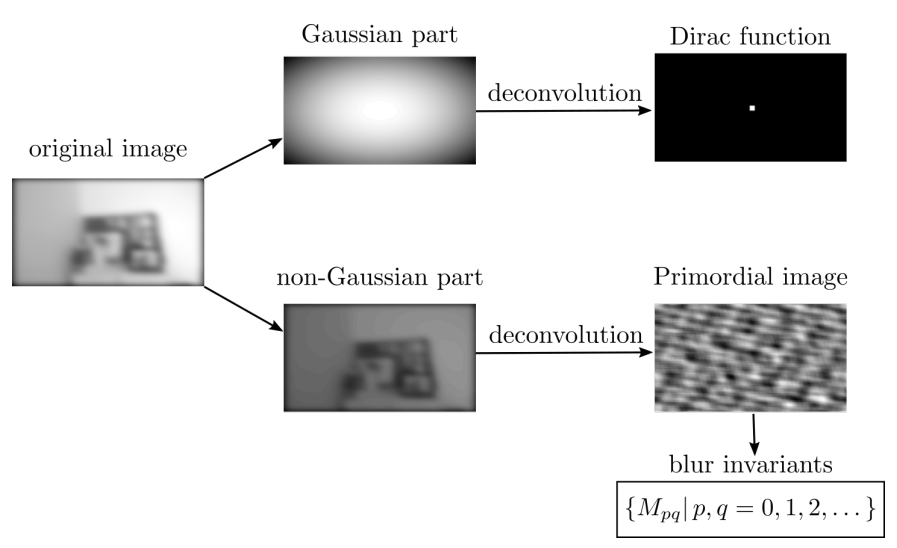

Fig. 1. The main idea of the blur invariants: The image is projected onto a set of Gaussians and this projection (i.e. the Gaussian part of the image) is used to "deconvolve" the image in Fourier domain. Blur-invariant primordial image is obtained as the result of this operation. Moments of the primordial image are blur invariants introduced in Eq. (8).

\section{COMBINED INVARIANTS}

The design of the combined affine-blur invariants is only possible because $S$ is closed to both Gaussian blur and affine transform, see Proposition 1. These two image degradations are commutative since $(f * h)^{\prime}=1 /\|A\|\left(f^{\prime} * h^{\prime}\right)$ and still $h^{\prime} \in S$. If the covariance matrix of the blur was constrained to be diagonal, such as in [8] and all other earlier papers, 
we could never combine the blur invariance and the affine invariance together. This is why the combined invariants have not been constructed yet.

The key idea of designing the combined invariants follows from the fact that the blur invariants $M_{p q}$ in Eq. (8) can be interpreted as moments of the primordial image $f_{r}$. So we can simply substitute them into any affine or rotation moment invariant and we end up with a combined invariant. This strong result is summarized in the following Theorem.

Theorem 1 (Substitution Theorem). Let $f$ be an image function and let $M_{p q}$ be invariants w.r.t. Gaussian blur defined by $E q$. (8). Let $f^{\prime}(\mathbf{x})=f(A \mathbf{x})$, A being a regular $2 \times 2$ matrix. Let $J\left(m_{p q} \mid p, q=0, \ldots, r\right)$ be an absolute invariant of image moments w.r.t. A, i.e. $J\left(m_{p q}^{\prime} \mid p, q=0, \ldots, r\right)=J\left(m_{p q} \mid p, q=\right.$ $0, \ldots, r)$. Then $J\left(M_{p q} \mid p, q=0, \ldots, r\right)$ is a relative invariant w.r.t. both $A$ and Gaussian blur as

$$
\|A\|^{w} J\left(M_{p q}^{\prime} \mid p, q=0, \ldots, r\right)=J\left(M_{p q} \mid p, q=0, \ldots, r\right),
$$

where $w$ is the weight ${ }^{1}$ of invariant $J\left(m_{p q}\right)$.

Proof. The proof follows the scheme shown in Fig. 2. First we show how the primordial image is transformed if the original image has undergone an affine transform $f^{\prime}(\mathbf{x})=f(A \mathbf{x})$. Using basic properties of Fourier transform, we have

$$
I\left(f^{\prime}\right)(\mathbf{u})=I(f)\left(A^{-T} \mathbf{u}\right) .
$$

Applying inverse Fourier transform, we obtain

$$
f_{r}^{\prime}(\mathbf{x})=\|A\| f_{r}(A \mathbf{x}),
$$

where $f_{r}^{\prime}$ is the primordial image of $f^{\prime}$. This relation tells us that the primordial image is transformed by the same coordinate transformation (up to a factor $\|A\|$ ) as the original image.

The moments $M_{p q}^{\prime}$ of $f_{r}^{\prime}(\mathbf{x})$ are related to the moments $\tilde{M}_{p q}$ of $f_{r}(A \mathbf{x})$ as $M_{p q}^{\prime}=\|A\| \tilde{M}_{p q}$ for any $p$ and $q$. In the theory of affine moment invariants [10], [4], it is well known that any absolute invariant $J\left(m_{p q} \mid p, q=0, \ldots, r\right)$ must have a form of a finite sum, where all terms are products of $K$ moments ( $K$ is called the degree of the invariant) divided by the $(K+w)$-th power of $m_{00}$. The statement of Theorem 1 follows immediately from this fact. Note that the invariance of $J\left(M_{p q} \mid p, q=0, \ldots, r\right)$ w.r.t. Gaussian blur is obvious and does not depend on the order in which the blurring and the coordinate transformation $A$ have been applied.

Since $A$ is usually unknown in practice, absolute invariants are more convenient image descriptors than the relative ones. An absolute combined invariant can be obtained as a ratio of two relative invariants of the same weight or, more generally, as a ratio of any two products of various relative invariants such that the factor $\|A\|$ is cancelled.

\footnotetext{
${ }^{1}$ The term weight of an invariant has been commonly used in the theory of algebraic invariants, see for instance [10], [4] for the definition. For any given invariant, its weight is known and follows from the way how the invariant has been constructed.
}

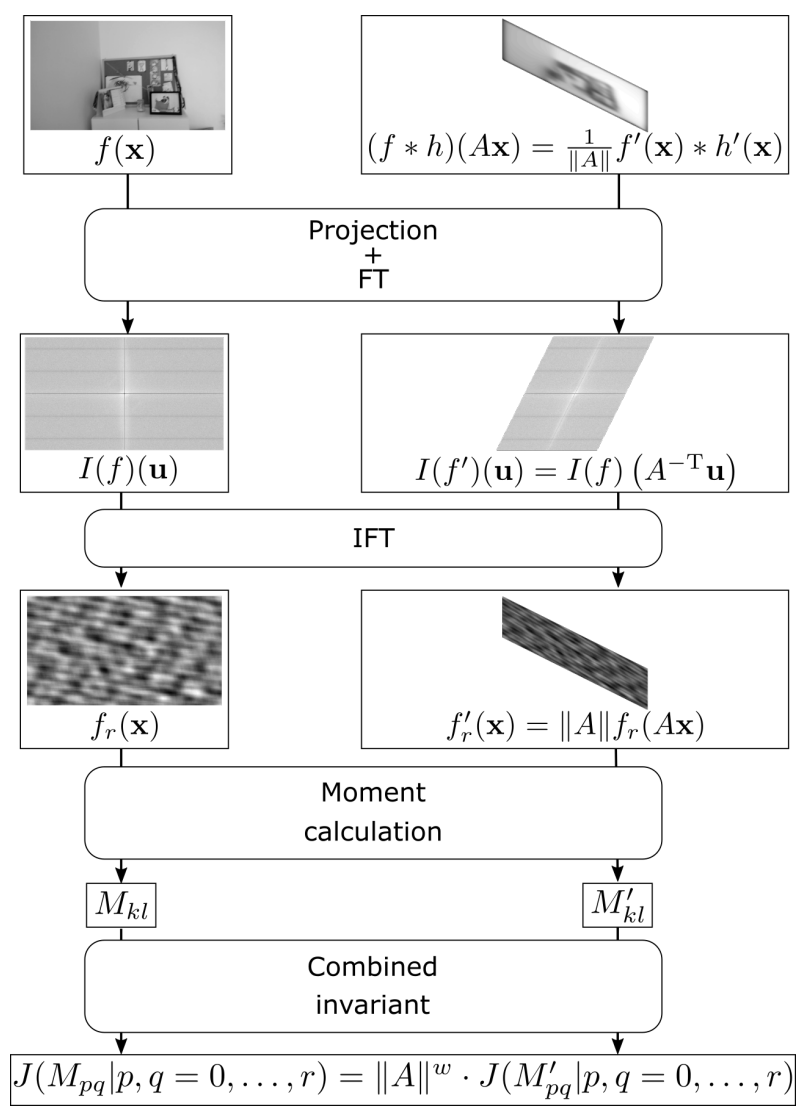

Fig. 2. Visual explanation of the Substitution Theorem. In practice, the combined invariant $J\left(M_{p q} \mid p, q=0, \ldots, r\right)$ is computed directly from image $f$ without the intermediate steps.

\section{NUMERICAL EXPERIMENTS}

In this Section, we examine the properties of two kinds of the combined invariants, both designed according to Theorem 1. First, the classical rotation moment invariants [11] up to the 8 th order were used as $J\left(m_{p q}\right)$. In the second case we employed affine moment invariants (AMIs) [10] as $J\left(m_{p q}\right)$ to get combined affine-blur invariants.

\section{A. Verification of the invariance}

To demonstrate the invariance property, we choose a template shown in Figure 3. First, we generated 100 random degradations (combination of rotation and Gaussian blur). Relative errors of 16 invariants invariants up to the 7 th order are shown in Figure 4. Only small errors due to resampling and the boundary effect (this occurs when the blurring mask goes across the template border) can be observed, which clearly demonstrates the invariance to rotation and blur.

We repeated the experiment using random affine transformations and 22 affine invariants up to the maximum weight 8 . Relative errors of the invariants are shown in Figure 5. The errors are slightly higher than for the rotation invariants because of higher influence of resampling errors. Still, the majority of errors is bellow $1 \%$. 


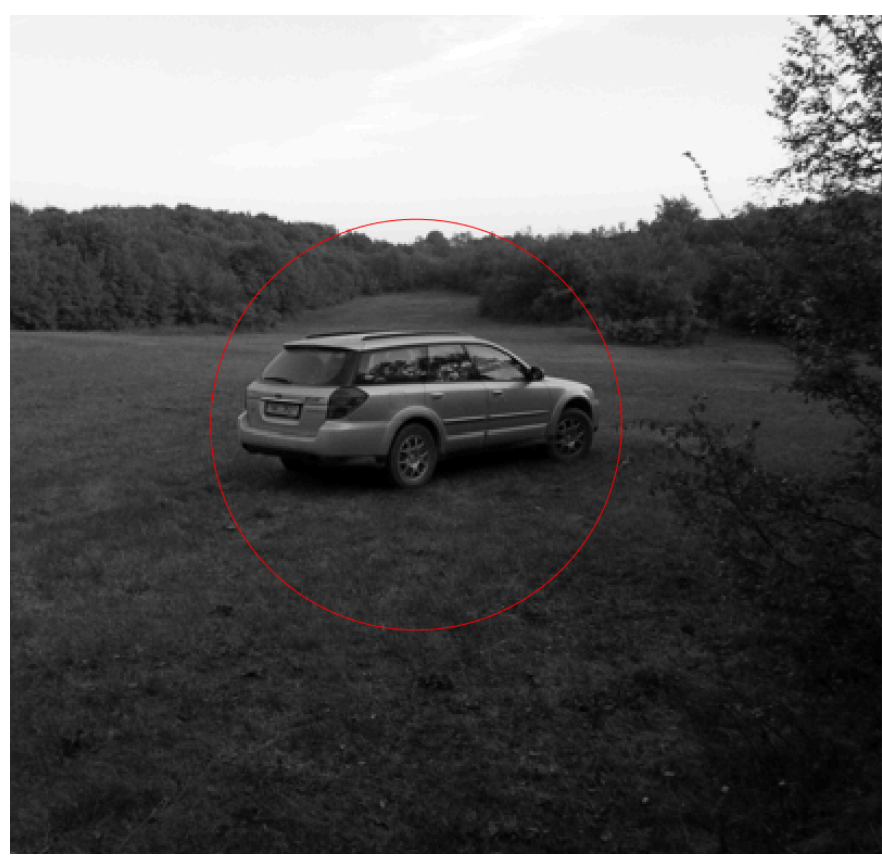

Fig. 3. The template used in the invariance test.

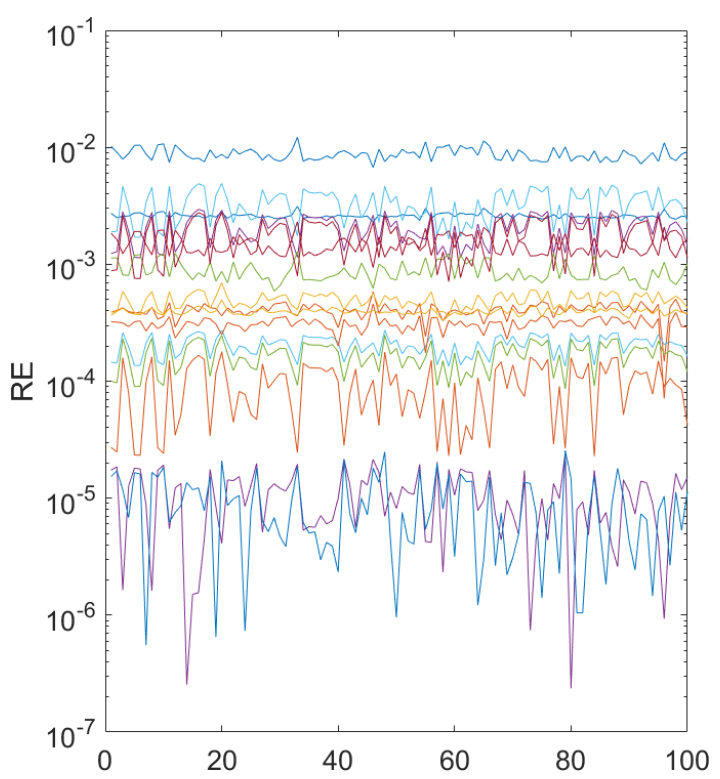

Fig. 4. Relative errors (vertical axis) of rotation invariants up to the 7 th order for 100 randomly generated rotations and blurs (horizontal axis).

\section{B. Robustness test}

In this experiment, we tested the robustness of the proposed features w.r.t. additive white Gaussian noise. For each SNR from $-5 \mathrm{~dB}$ to $80 \mathrm{~dB}$ with the step of $1 \mathrm{~dB}$, we generated 100 realizations of the noisy image. We computed the mean relative errors of all invariants and the standard deviations. The results are summarized in Figure 6. We can see that all invariants are sufficiently stable. This can be expected because the moments, as integral features, tend to average out the impact of the noise. Slightly higher robustness of the affine invariants at low SNR

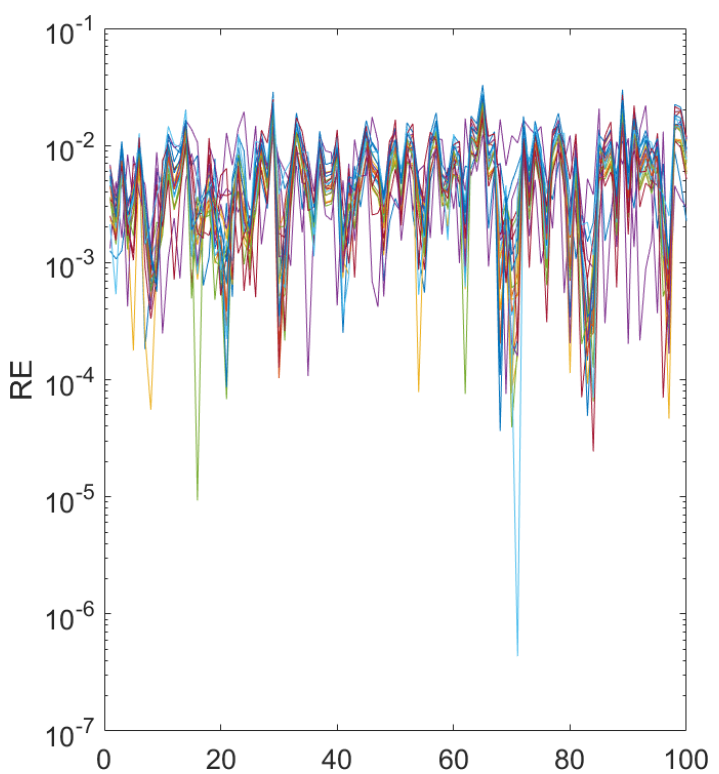

Fig. 5. Relative errors (vertical axis) of affine invariants up to maximal weight 8 for 100 randomly generated affine transformations and blurs (horizontal axis).

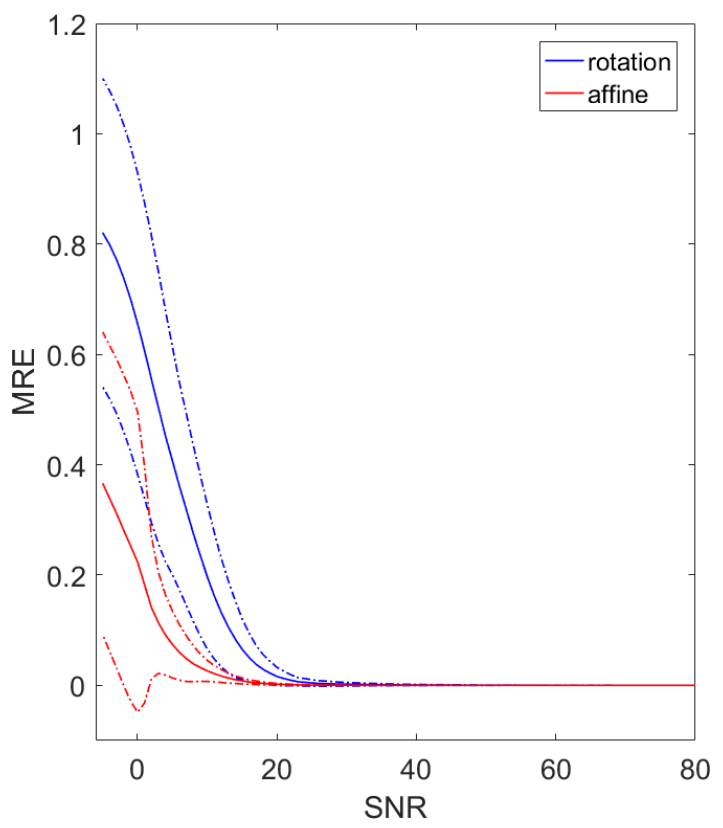

Fig. 6. Mean relative error of rotation invariants (solid blue line) \pm standard deviation (dotdash blue line) and mean relative error of affine invariants (solid red line) \pm standard deviation (dotdash red line) for SNR from $-5 \mathrm{~dB}$ to 80 dB.

is probably because these invariants are composed of several terms and we witness an error cancellation effect.

\section{Matching of blurred and rotated templates}

In this experiment, we tested the use of the combined blur-rotation invariants in template matching. As a reference method for a comparison, we used blur invariants w.r.t. circular Gaussian blur from [8] substituted in the same rotation invariants. 


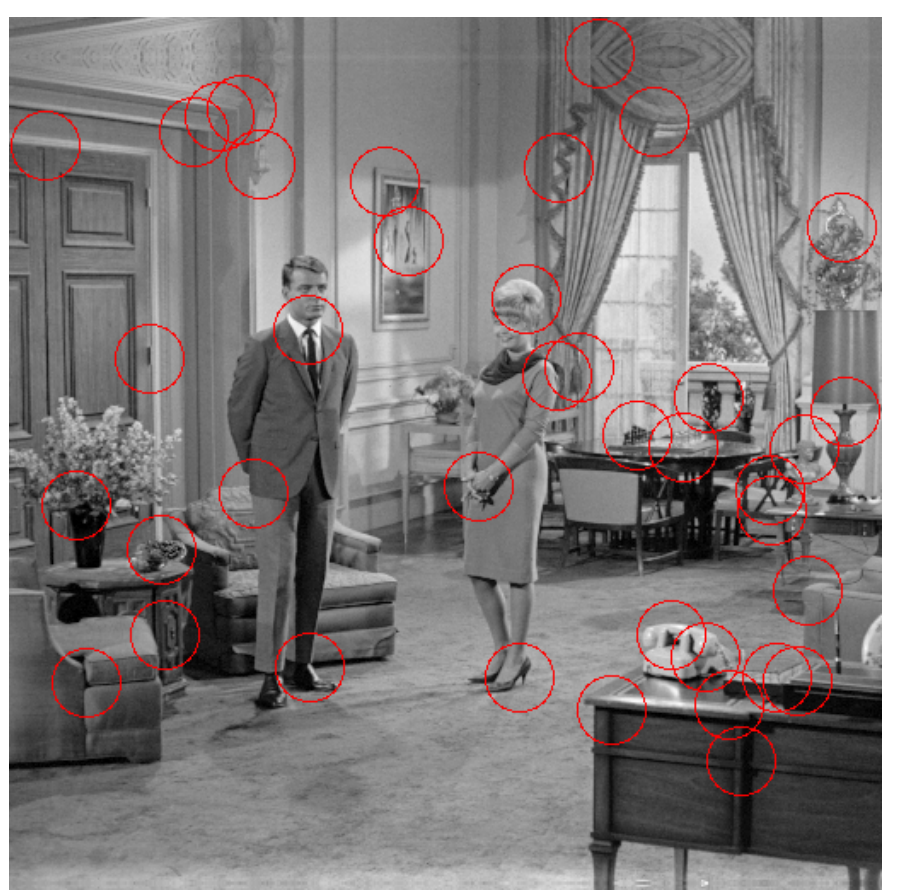

Fig. 7. The picture of a living room with the selected templates.

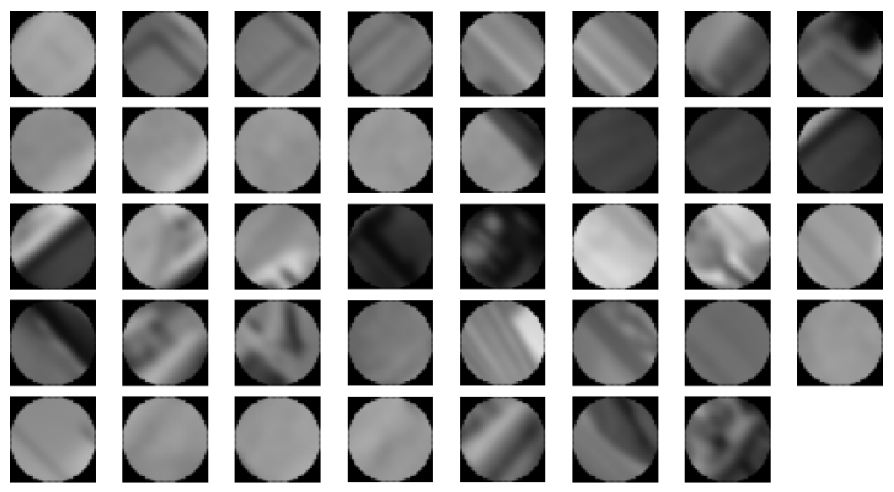

Fig. 8. Blurred and rotated templates.

From the clear test image, we extracted 39 circular templates which contain dominant structures such as edges or corners (see Fig. 7). We rotated each template, blurred them by an anisotropic Gaussian blur, and matched them against the original ones (see Fig. 8 for the degraded templates). The matching was performed by searching for the minimum $\ell_{2}$ distance in the space of the combined invariants. We run the experiment six times with maximal order of the invariants ranging from three to eight. The results are summarized in Table I.

The proposed combined invariants perform well for the maximum order greater than three (the 3rd-order invariants apparently do not capture enough discriminative information about the templates) and perform significantly better than the competitor. This is because the invariants from [8] cannot handle appropriately the anisotropic blur.

\begin{tabular}{c||c|c|c|c|c|c} 
Maximum moment order & 3 & 4 & 5 & 6 & 7 & 8 \\
\hline \hline \# of errors (proposed method) & 9 & 1 & 0 & 0 & 0 & 1 \\
\# of errors (method from [8]) & 33 & 19 & 14 & 16 & 16 & 16
\end{tabular}
TABLE I

MATCHING ERRORS (OUT OF 39 TRIALS) OF THE PROPOSED COMBINED INVARIANTS AND OF ANALOGOUS COMBINED INVARIANTS FROM [8].

\section{Classification of blurred and affinely deformed digits}

The last experiment demonstrates the recognition power of the proposed combined affine-blur invariants. For this test, we used the popular MNIST dataset of handwritten digits [12]. For each digit $0,1, \ldots, 9$ we randomly generated 50 blurred and affinely deformed instances (see Fig. 9 for some examples) and classified them against the original dataset.

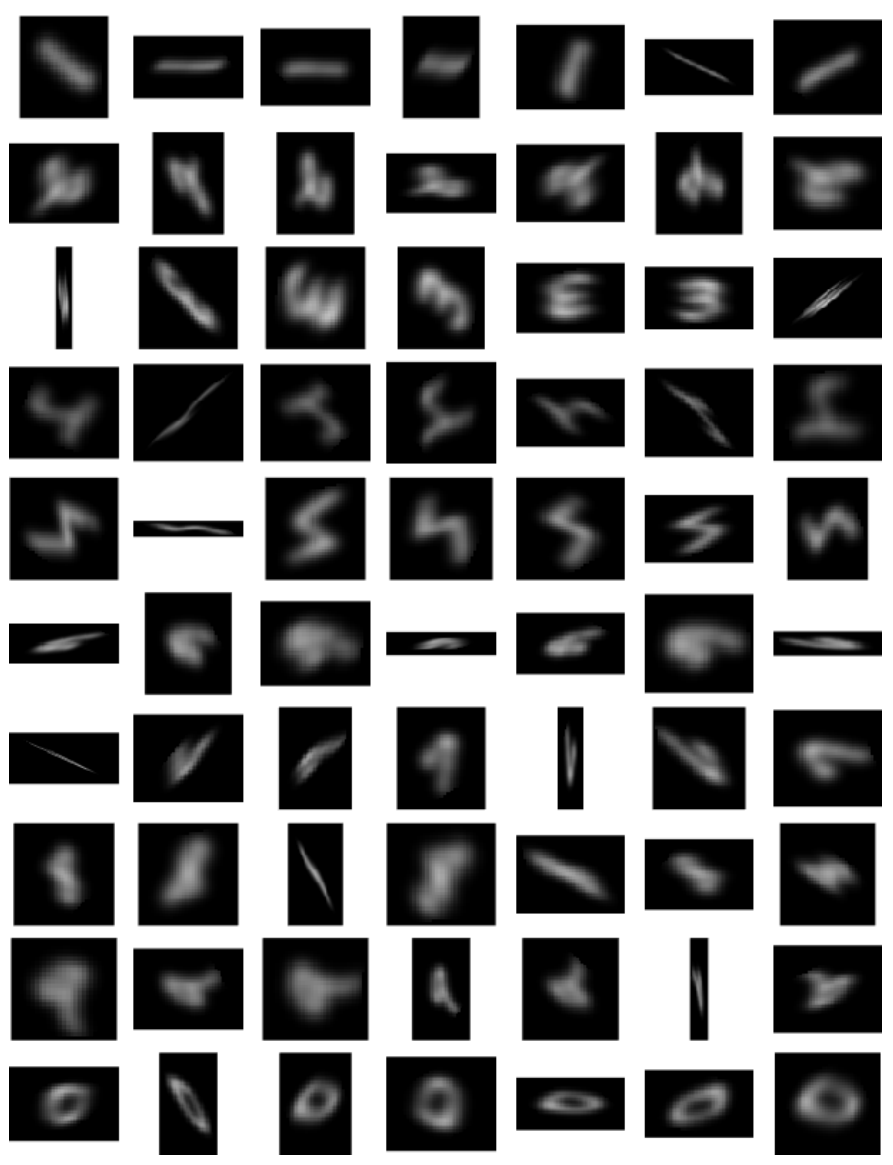

Fig. 9. Examples of blurred and deformed digits.

To illustrate the advantage of the combined invariants, we compared them both to "pure" AMIs [10] and to "pure" Gaussian blur invariants (8). The combined invariants yielded the overall recognition rate $98.4 \%$, while the AMIs only $20 \%$ and the blur invariants performed even worse yielding approximately $15 \%$ success rate. This clearly shows that the Substitution Theorem brings invariants of a new quality.

\section{GeneralizATION}

Possible extension of the theory presented in this paper to images whose spatial coordinates are from 3D (or even 
higher) space depends basically on three factors. The first is an extension of Gaussian blur invariants from (8), the second one is the validity of the Substitution Theorem, and the last requirement is the existence of appropriate geometric invariants (affine or at least rotational) that could be used in the Substitution Theorem as $J$. Although it was not discussed in [1], the first requirement is relatively simply to fulfill. The definition and all properties of $I(f)$ do not depend on the dimension. We can even repeat the Taylor expansion in terms of moments. Transition to the form analogous to Eq. (8) depends on our ability to compute $d$-dimensional moments of a Gaussian. For $d>2$, this is extremely laborious but possible.

The Substitution Theorem is formally valid for any $d$. However, combined invariants are of practical interest only for $d=2$ and $d=3$. We can use the sets of 3D rotation moment invariants [13], [14] or 3D affine invariants [15], [16] and substitute into them the 3D analogue of Eq. (8). For $d>3$, there have not been studied/published any moment invariants w.r.t. $A$, so in fact we do not have formulas which we could substitute in.

Finding blur invariants to parametric kernels other than Gaussian is probably impossible in the moment domain. The necessary (but not sufficient) condition for such kernels is their closure w.r.t. convolution. Surprisingly, there are very few parametric families of functions which are closed under convolution. Even the class of 1D generalized Gaussian distributions [17], where one might intuitively expect the closure property, is not closed under convolution for exponents other than two.

The functions with the closure convolution property are known as alpha-stable distributions. Only very few of them have a closed-form formula expressible in terms of elementary functions (Gaussian, Cauchy, and Lévy distributions). Among all alpha-stable functions, only the Gaussian has finite moments of all orders. This leads to the conclusion that moment invariants w.r.t. convolution with an alpha-stable parametric kernel do not exist for other than Gaussian kernels. ${ }^{2}$

\section{CONCLUSION}

We proposed new invariants w.r.t. anisotropic Gaussian blur and affine transformation of the image. Although they were derived by means of projection operators and Fourier transform, they can be calculated directly in the image domain, without an explicit construction of the projections. The invariance to an affine transform is achieved thanks to the proposed Substitution Theorem, which is the main result of the paper. Experimental evaluation showed a good performance of the invariants. However, they are not suitable in all cases and acquisition scenarios as they suffer from the common limitations of all moment invariants. Being intrinsically global, they are calculated from the entire image/template. Local image deformations and partial occlusions would affect all invariants and would make them unstable. This can be partially

\footnotetext{
${ }^{2}$ There is, however, still a possibility of finding blur invariants in the Fourier domain using a parametric form of their characteristic functions.
}

overcame by weighting functions that emphasize the regions of interest but it does not resolve the problem in principle.

In the future work, it would be interesting to couple the proposed blur-invariant representation with the CNNs in order to make the CNNs blur-invariant without any data augmentation and/or geometric normalization.

\section{ACKNOWLEDGMENT}

This work has been supported by the Czech Science Foundation under the grant No. GA18-07247S, by the Grant SGS18/188/OHK4/3T/14 provided by the Ministry of Education, Youth, and Sports of the Czech Republic (MŠMT ČR), and by the Praemium Academiae.

\section{REFERENCES}

[1] J. Kostková, J. Flusser, M. Lébl, and M. Pedone, "Image invariants to anisotropic Gaussian blur," in Proceedings of the Scandinavian Conference on Image Analysis SCIA'19, vol. LNCS 11482. Springer, 2019, pp. 140-151.

[2] J. Flusser, T. Suk, and S. Saic, "Recognition of blurred images by the method of moments," IEEE Transactions on Image Processing, vol. 5, no. 3, pp. 533-538, 1996.

[3] J. Flusser and T. Suk, "Degraded image analysis: An invariant approach," IEEE Transactions on Pattern Analysis and Machine Intelligence, vol. 20, no. 6, pp. 590-603, 1998.

[4] J. Flusser, T. Suk, and B. Zitová, 2D and 3D Image Analysis by Moments. Chichester, U.K.: Wiley, 2016.

[5] J. Liu and T. Zhang, "Recognition of the blurred image by complex moment invariants," Pattern Recognition Letters, vol. 26, no. 8, pp. $1128-1138,2005$.

[6] B. Xiao, J.-F. Ma, and J.-T. Cui, "Combined blur, translation, scale and rotation invariant image recognition by Radon and pseudo-FourierMellin transforms," Pattern Recognition, vol. 45, pp. 314-321, 2012.

[7] C. Höschl IV and J. Flusser, "Robust histogram-based image retrieval," Pattern Recognition Letters, vol. 69, no. 1, pp. 72-81, 2016.

[8] J. Flusser, S. Farokhi, C. Höschl IV, T. Suk, B. Zitová, and M. Pedone, "Recognition of images degraded by Gaussian blur," IEEE Transactions on Image Processing, vol. 25, no. 2, pp. 790-806, 2016.

[9] Z. Zhang, E. Klassen, and A. Srivastava, "Gaussian blurring-invariant comparison of signals and images," IEEE Transactions on Image Processing, vol. 22, no. 8, pp. 3145-3157, 2013.

[10] T. Suk and J. Flusser, "Affine moment invariants generated by graph method," Pattern Recognition, vol. 44, no. 9, pp. 2047-2056, 2011.

[11] J. Flusser, "On the independence of rotation moment invariants," Pattern Recognition, vol. 33, no. 9, pp. 1405-1410, 2000.

[12] Y. LeCun and C. Cortes, "MNIST handwritten digit database," 2010. [Online]. Available: http://yann.lecun.com/exdb/mnist/

[13] F. A. Sadjadi and E. L. Hall, "Three dimensional moment invariants," IEEE Transactions on Pattern Analysis and Machine Intelligence, vol. 2, no. 2, pp. 127-136, 1980.

[14] T. Suk and J. Flusser, "Tensor method for constructing 3D moment invariants," in Computer Analysis of Images and Patterns CAIP'11, ser. Lecture Notes in Computer Science, P. Real, D. Diaz-Pernil, H. MolinaAbril, A. Berciano, and W. Kropatsch, Eds., vol. 6854-6855. Berlin, Heidelberg, Germany: Springer, August 2011, pp. 212-219.

[15] A. G. Mamistvalov, " $n$-dimensional moment invariants and conceptual mathematical theory of recognition $n$-dimensional solids," IEEE Transactions on Pattern Analysis and Machine Intelligence, vol. 20, no. 8, pp. 819-831, 1998.

[16] D. Xu and H. Li, "3-D affine moment invariants generated by geometric primitives," in Proceedings of the 18th International Conference on Pattern Recognition ICPR'06. Los Alamitos, California, USA: IEEE Computer Society, 2006, pp. 544-547.

[17] S. Nadarajah, "A generalized normal distribution," Journal of Applied Statistics, vol. 32, no. 7, pp. 685-694, 2005. 